\title{
Twisted mass lattice QCD with non-degenerate quark masses
}

\author{
Gernot Münster and Tobias Sudmann \\ Institut für Theoretische Physik, Universität Münster \\ Wilhelm-Klemm-Str. 9, D-48149 Münster, Germany \\ e-mail: munsteg@uni-muenster.de,tobisud@uni-muenster.de
}

March 22, 2006 (revised April 14, 2006)

\begin{abstract}
Quantum Chromodynamics on a lattice with Wilson fermions and a chirally twisted mass term is considered in the framework of chiral perturbation theory. For two and three numbers of quark flavours, respectively, with non-degenerate quark masses the pseudoscalar meson masses and decay constants are calculated in next-to-leading order including lattice effects quadratic in the lattice spacing $a$.
\end{abstract}

When quantum field theories are simulated numerically, the introduction of a finite space-time lattice brings with it two standard problems. In order to obtain results relevant to the physics in the infinite continuum the effects of the finite lattice spacing $a$ and of the finite volume of the system have to be controlled. The resulting demands on the lattice are limited by the available computer resources. Simulations of Quantum Chromodynamics are facing a third difficulty. The common algorithms slow down severely when the masses of the light quarks are decreased towards their physical values.

Recent investigations [1, 2, 3] suggest that lattice QCD with Wilson fermions and a chirally twisted quark mass term [4, 5] offers an attractive framework for numerical simulations with small up- and down-quark masses. In particular, for the case of full twist lattice artifacts of order $a$ are removed automatically [6]. For a review of twisted mass lattice QCD see [7]. 
An important tool for QCD is chiral perturbation theory [8, 9, 10]. For sufficiently small quark masses it describes the dependence of mesonic quantities like masses and decay constants on the quark masses in terms of expansions in powers of quark masses (modified by logarithms). These expansions contain low energy parameters, the Gasser-Leutwyler coefficients, which have to be determined by methods outside chiral perturbation theory.

Chiral perturbation theory is particularly suited for lattice QCD. In its range of applicability, it can be used to extrapolate the results of Monte Carlo simulations into the region of small quark masses. On the other hand, numerical simulations of lattice QCD can provide information about the Gasser-Leutwyler coefficients, see [11] for a review.

The adaption of chiral perturbation theory to lattice QCD [12, 13, 14, 15, 16] brings the lattice spacing $a$ in as a second expansion parameter. In the effective chiral Lagrangian lattice artifacts are represented by additional terms proportional to powers of $a$ [14. Physical quantities appear in double expansions in quark masses and in $a$.

Chiral perturbation theory has been extended to the case of twisted mass lattice QCD in [17, 18. The resulting expansions contain the twist angle as an additional parameter. Results for various quantities have been calculated in next-to-leading order of chiral perturbation theory including lattice effects of order $a^{2}$ [17, 18, 19, 20, 21, 22, 23, 24].

In most of the work the case of degenerate quark masses is considered. Current numerical work on twisted mass lattice QCD with non-degenerate quark masses [25] motivates to implement this situation in chiral perturbation theory. This is the purpose of the work presented here.

We consider lattice QCD with $N_{f}=2$ or 3 quark flavours and a twisted mass term. In the two-flavour case we consider split quark masses $\left(m_{u} \neq m_{d}\right)$. In the case of three flavours we restrict ourselves in this letter to equal up- and down-quark masses and a heavier strange quark mass $\left(m_{u}=m_{d} \neq m_{s}\right)$. The masses and decay constants of the pseudoscalar mesons are calculated in next-to-leading (one-loop) order, including lattice terms of order $a^{2}$.

Chiral perturbation theory for pseudoscalar meson masses in untwisted lattice QCD with $N_{f}=3$ flavours of Wilson quarks has been considered in [26]. These authors use a power counting scheme different from the one of [14, 16, 17, 18] and a different parameterization of the action, which hampers a comparison of our calculations. The results which could be compared in the limit of vanishing twist angle do agree. The case of twisted mass lattice QCD with $2+2$ flavours has been studied recently by means of chiral perturbation theory in [27]. The implementation of mass non-degenerate quarks in twisted mass chiral perturbation theory for two flavours has been considered by Walker-Loud and $\mathrm{Wu}$ [28]. They extend the formalism to the baryon sector and calculate nucleon and delta masses.

In lattice QCD a chiral twist of the quark mass term is generally introduced by transforming the quark mass matrix $M$ into

$$
M(\omega)=\mathrm{e}^{\mathrm{i} \omega_{b} T_{b} \gamma_{5}} M \mathrm{e}^{\mathrm{i} \omega_{b} T_{b} \gamma_{5}},
$$


where $T_{b}$ are the generators of $\mathrm{SU}\left(N_{f}\right)$ and $\omega$ denotes the set of group parameters $\omega_{b}$. In contrast to the situation in the continuum, on the lattice the chiral transformation of the mass matrix has a measurable effect on observables since in lattice QCD with Wilson fermions chiral symmetry is broken by lattice artifacts.

In chiral perturbation theory the low energy regime of QCD is described by an effective Lagrangian for the pseudo-Goldstone bosons of spontaneously broken chiral symmetry. For $N_{f}=3$ they form the pseudoscalar meson octet including the pions, kaons and $\eta$.

The transformation properties of the Goldstone bosons in QCD lead to collect the dynamical variables in the $\mathrm{SU}\left(N_{f}\right)$ matrix valued field

$$
U(x)=\exp \left(2 \frac{\mathrm{i}}{F_{0}} T_{b} \phi_{b}(x)\right),
$$

where $\phi_{b}$ are the components of the meson fields and $F_{0}$ is a low energy parameter. Treating quark masses and lattice effects perturbatively in a spurion analysis with respect to the required symmetry properties, the leading order effective chiral Lagrangian for lattice QCD is given by $[12,14$

$$
\mathcal{L}_{\mathrm{LO}} \equiv \mathcal{L}_{2}=\frac{F_{0}^{2}}{4} \operatorname{Tr}\left(\partial_{\mu} U \partial_{\mu} U^{\dagger}\right)-\frac{F_{0}^{2}}{4} \operatorname{Tr}\left(\chi U^{\dagger}+U \chi^{\dagger}\right)-\frac{F_{0}^{2}}{4} \operatorname{Tr}\left(\rho U^{\dagger}+U \rho^{\dagger}\right),
$$

consisting of the chiral symmetric kinetic term and two chiral symmetry breaking terms. The first of them contains the quark mass term, given by

$$
\chi=2 B_{0} M
$$

and in the second one lattice artifacts are represented by

$$
\rho=2 W_{0} a 1
$$

where $B_{0}$ and $W_{0}$ are additional parameters.

Note that we use the power-counting scheme of [14, 16], accounting for terms of order $\{m, a\}$ in leading order and $\left\{m^{2}, m a, a^{2}\right\}$ in next-to-leading order. In order to define an applicable scheme we always assume the lattice effects to be smaller: $W_{0} a \ll B_{0} m_{q}$.

Introducing a chiral twist, the mass matrix in the effective Lagrangian becomes

$$
\chi(\omega)=2 B_{0} \mathrm{e}^{-\mathrm{i} \omega_{b} T_{b}} M \mathrm{e}^{-\mathrm{i} \omega_{b} T_{b}},
$$

in the so-called twisted basis. The $\omega_{b}$-dependence of the mass term can be removed by changing to the physical basis, i.e. by a chiral transformation of the configuration matrix

$$
U \longrightarrow U^{\prime}=\mathrm{e}^{\mathrm{i} \omega_{b} T_{b}} U \mathrm{e}^{\mathrm{i} \omega_{b} T_{b}}
$$

and a corresponding chiral twist of the lattice term according to

$$
\rho \longrightarrow \rho(\omega)=\mathrm{e}^{\mathrm{i} \omega_{b} T_{b}} \rho \mathrm{e}^{\mathrm{i} \omega_{b} T_{b}} .
$$


Omitting the prime on the meson field and suppressing the explicit $\omega_{b}$-dependence of the lattice term, the effective Lagrangian looks like the one of untwisted lattice QCD. However, the introduction of a chirally twisted mass term leads to a shift of the minimum $\tilde{\phi} \neq 0$ of the effective Lagrangian of order $a$. Chiral perturbation theory, which is based on an expansion around this minimum, has to be adapted accordingly. We treat the nontrivial minimum perturbatively according to the applied scheme. Denoting the minimum

$$
U_{0}=\exp \left(2 \frac{\mathrm{i}}{F_{0}} T_{b} \tilde{\phi}_{b}\right),
$$

a substitution

$$
U \longrightarrow U_{0}^{1 / 2} U U_{0}^{1 / 2}
$$

is performed before expanding the Lagrangian. The tree-level and one-loop contributions of the resulting $\mathcal{L}_{2}$ have the same form as those of continuum chiral perturbation theory, but with different leading order meson masses which include lattice artifacts, see below.

The next-to-leading order part of the effective Lagrangian in the present power counting scheme is given by [16, 17]

$$
\begin{aligned}
\mathcal{L}_{4}= & -L_{1}\left[\operatorname{Tr}\left(\partial_{\mu} U \partial_{\mu} U^{\dagger}\right)\right]^{2}-L_{2}\left[\operatorname{Tr}\left(\partial_{\mu} U \partial_{\nu} U^{\dagger}\right)\right]^{2}-L_{3} \operatorname{Tr}\left(\left[\partial_{\mu} U \partial_{\mu} U^{\dagger}\right]^{2}\right) \\
& +L_{4} \operatorname{Tr}\left(\partial_{\mu} U \partial_{\mu} U^{\dagger}\right) \operatorname{Tr}\left(\chi^{\dagger} U+U^{\dagger} \chi\right)+W_{4} \operatorname{Tr}\left(\partial_{\mu} U \partial_{\mu} U^{\dagger}\right) \operatorname{Tr}\left(\rho^{\dagger} U+U^{\dagger} \rho\right) \\
& +L_{5} \operatorname{Tr}\left(\partial_{\mu} U \partial_{\mu} U^{\dagger}\left(U \chi^{\dagger}+\chi U^{\dagger}\right)\right)+W_{5} \operatorname{Tr}\left(\partial_{\mu} U \partial_{\mu} U^{\dagger}\left(U \rho^{\dagger}+\rho U^{\dagger}\right)\right) \\
& -L_{6}\left[\operatorname{Tr}\left(\chi^{\dagger} U+U^{\dagger} \chi\right)\right]^{2}-W_{6} \operatorname{Tr}\left(\chi^{\dagger} U+U^{\dagger} \chi\right) \operatorname{Tr}\left(\rho^{\dagger} U+U^{\dagger} \rho\right)-W_{6}^{\prime}\left[\operatorname{Tr}\left(\rho^{\dagger} U+U^{\dagger} \rho\right)\right]^{2} \\
& -L_{7}\left[\operatorname{Tr}\left(\chi^{\dagger} U-U^{\dagger} \chi\right)\right]^{2}-W_{7} \operatorname{Tr}\left(\chi^{\dagger} U-U^{\dagger} \chi\right) \operatorname{Tr}\left(\rho^{\dagger} U-U^{\dagger} \rho\right)-W_{7}^{\prime}\left[\operatorname{Tr}\left(\rho^{\dagger} U-U^{\dagger} \rho\right)\right]^{2} \\
& -L_{8} \operatorname{Tr}\left(\chi^{\dagger} U \chi^{\dagger} U+U^{\dagger} \chi U^{\dagger} \chi\right)-W_{8} \operatorname{Tr}\left(\rho^{\dagger} U \chi^{\dagger} U+U^{\dagger} \rho U^{\dagger} \chi\right) \\
& -W_{8}^{\prime} \operatorname{Tr}\left(\rho^{\dagger} U \rho^{\dagger} U+U^{\dagger} \rho U^{\dagger} \rho\right) .
\end{aligned}
$$

Next-to-leading order terms are given by tree-level contributions from $\mathcal{L}_{4}$ and one-loop contributions from $\mathcal{L}_{2}$. The latter produce divergencies, which are treated by renormalizing the low energy coefficients $L_{i}, W_{i}$ and $W_{i}^{\prime}$.

First we consider $N_{f}=2$ flavours with non-degenerate quark masses. Following [29], to ensure positivity of the fermion determinant we implement the quark mass splitting and the chiral twist with different generators $T_{b}$. Because $T_{3}=\tau_{3} / 2$ is usually chosen for the twist, we use $T_{1}=\tau_{1} / 2$ for the mass difference. Defining

$$
\chi_{0}=B_{0}\left(m_{d}+m_{u}\right) \quad \text { and } \quad \chi_{1}=B_{0}\left(m_{d}-m_{u}\right)
$$

we have

$$
\chi=\chi_{0} 1+\chi_{1} \tau_{1}
$$

and

$$
\rho=\rho_{0} \mathbb{1}+\mathrm{i} \rho_{3} \tau_{3}
$$


where $\rho_{0}=2 W_{0} a \cos \omega$ and $\rho_{3}=2 W_{0} a \sin \omega$. Due to the properties of the Pauli matrices only the $L_{7}$ and $L_{8}$-terms lead to contributions involving the mass splitting $\chi_{1}$.

For the minimum of the effective Lagrangian we obtain

$$
\tilde{\phi}_{1}=\tilde{\phi}_{2}=0
$$

and

$$
\tilde{\phi}_{3}=F_{0} \frac{\rho_{3}}{\chi_{0}}\left[1-\frac{8}{F_{0}^{2}}\left(2 L_{86}-W_{86}\right) \chi_{0}\right]+\mathcal{O}\left(a^{2}\right),
$$

where terms of order $a^{2}$ can be neglected at this order of the calculation, and we define

$$
L_{i j}=L_{i}+N_{f} L_{j}
$$

From a one-loop calculation of the renormalized propagator we obtain the pion masses in next-to-leading order. Owing to the unequal quark masses and the chiral twist the three masses are generally different. For the component $\phi_{2} \equiv \pi_{2}$ we get

$$
\begin{aligned}
M_{\pi_{2}}^{2}=m_{\pi}^{2} & +\frac{m_{\pi}^{4}}{32 \pi^{2} F_{0}^{2}} \ln \left(\frac{m_{\pi}^{2}}{\Lambda^{2}}\right) \\
& +\frac{8}{F_{0}^{2}}\left[-L_{54}^{r} \chi_{0}\left(\chi_{0}+\rho_{0}\right)-W_{54}^{r}\left(\chi_{0} \rho_{0}+\rho_{0}^{2}+\rho_{3}^{2}\right)\right. \\
& \left.+2 L_{86}^{r}\left(\chi_{0}^{2}-\rho_{3}^{2}\right)+2 W_{86}^{r}\left(\chi_{0} \rho_{0}+\rho_{3}^{2}\right)+2 W_{86}^{\prime r} \rho_{0}^{2}\right],
\end{aligned}
$$

where $m_{\pi}$ denotes the leading order pion mass,

$$
m_{\pi}^{2}=\chi_{0}+\rho_{0}+\frac{\rho_{3}^{2}}{2 \chi_{0}}
$$

$\Lambda$ is the renormalization scale, usually taken to be $4 \pi F_{0}$, and the superscript $r$ indicates the renormalized $L, W$ and $W^{\prime}$ coefficients.

The masses of the other two pions can be read of from the mass splittings

$$
\begin{aligned}
& M_{\pi_{1}}^{2}-M_{\pi_{2}}^{2}=\frac{16}{F_{0}^{2}} L_{87}^{r} \chi_{1}^{2} \\
& M_{\pi_{2}}^{2}-M_{\pi_{3}}^{2}=\frac{16}{F_{0}^{2}}\left(L_{86}^{r}-W_{86}^{r}+W_{86}^{\prime r}\right) \rho_{3}^{2} .
\end{aligned}
$$

Setting $\chi_{1}=0$, this is consistent with the results for degenerate quark masses [17, 20]. Moreover we observe $\mathcal{O}(a)$-improvement at maximum twist $\omega=\pi / 2$, where $\rho_{0}=0$.

Note that with our choice of $\mathrm{SU}(2)$ generators, $\pi_{1}$ is the neutral pion, and the charged pions are linear combinations of $\pi_{2}$ and $\pi_{3}$. Due to the twist, however, the mass eigenstates are not the usual charged pions but the components $\pi_{2}$ and $\pi_{3}$.

The pion decay constant $F_{\pi}$ is given by the matrix element

$$
\left\langle 0\left|A_{\mu, a}\right| \phi_{b}(\vec{p})\right\rangle=\mathrm{i} F_{\phi} p_{\mu} \delta_{a b}
$$


where $A_{\mu, a}$ is the axial current. In next-to-leading order one can determine the decay constant including order $a$ terms. We get

$$
\frac{F_{\pi}}{F_{0}}=1-\frac{1}{16 \pi^{2} F_{0}^{2}} m_{\pi}^{2} \ln \left(\frac{m_{\pi}^{2}}{\Lambda^{2}}\right)+\frac{4}{F_{0}^{2}}\left(L_{54}^{r} \chi_{0}+W_{54}^{r} \rho_{0}\right)
$$

without any contributions of the quark mass difference $\chi_{1}$ and coinciding with the result of [17.

Now we turn to the consideration of $N_{f}=3$ flavours with degenerate up- and down-quarks and a heavier strange-quark. The generators of SU(3) are given by the Gell-Mann matrices $T_{b}=\lambda_{b} / 2$. Defining $\chi_{s}=2 B_{0} m_{s}$, the quark mass matrix is given by

$$
\chi=\frac{1}{3}\left(2 \chi_{0}+\chi_{s}\right) \mathbb{1}-\frac{1}{\sqrt{3}}\left(\chi_{s}-\chi_{0}\right) \lambda_{8} .
$$

For $N_{f}=3$ it is generally possible to introduce a twist with two twist angles involving all flavours. In view of the fact that the motivation for twist is to eliminate infrared problems stemming from the light quark masses, and in order to ensure a positive quark determinant [30] we implement a chiral twist by a single angle $\omega$ in the isospin sector of the lightest quarks, leading to

$$
\rho=\left(\begin{array}{ccc}
\rho_{0}+\mathrm{i} \rho_{3} & 0 & 0 \\
0 & \rho_{0}-\mathrm{i} \rho_{3} & 0 \\
0 & 0 & |\rho|
\end{array}\right)=\frac{1}{3}\left(2 \rho_{0}+|\rho|\right) \mathbb{1}-\frac{1}{\sqrt{3}}\left(|\rho|-\rho_{0}\right) \lambda_{8}+\mathrm{i} \rho_{3} \lambda_{3}
$$

with $|\rho|=2 W_{0}$ a.

The minimum of the potential is shifted again in $T_{3}$-direction to

$$
\tilde{\pi}_{3}=F_{0} \frac{\rho_{3}}{\chi_{0}}\left[1-\frac{8}{F_{0}^{2}}\left\{\left(2 L_{8}-W_{8}\right) \chi_{0}+\left(2 L_{6}-W_{6}\right)\left(2 \chi_{0}+\chi_{s}\right)\right\}\right]+\mathcal{O}\left(a^{2}\right) .
$$

At leading order the pseudoscalar meson masses are

$$
\begin{aligned}
m_{\pi}^{2} & =\chi_{0}+\rho_{0}+\frac{\rho_{3}^{2}}{2 \chi_{0}}, \\
m_{K}^{2} & =\frac{1}{2}\left[\left(\chi_{s}+\chi_{0}\right)+\left(|\rho|+\rho_{0}\right)+\frac{\rho_{3}^{2}}{2 \chi_{0}}\right], \\
m_{\eta}^{2} & =\frac{1}{3}\left[\left(2 \chi_{s}+\chi_{0}\right)+\left(2|\rho|+\rho_{0}\right)+\frac{\rho_{3}^{2}}{2 \chi_{0}}\right] .
\end{aligned}
$$

Including the tree-level and one-loop terms at next-to-leading order we obtain the pion 
masses

$$
\begin{aligned}
M_{\pi_{1,2}}^{2}= & m_{\pi}^{2}+\frac{m_{\pi}^{2}}{96 \pi^{2} F_{0}^{2}}\left[3 m_{\pi}^{2} \ln \left(\frac{m_{\pi}^{2}}{\Lambda^{2}}\right)-m_{\eta}^{2} \ln \left(\frac{m_{\eta}^{2}}{\Lambda^{2}}\right)\right] \\
& +\frac{4}{F_{0}^{2}}\left[\left(4 L_{8}^{r}+8 L_{6}^{r}-2 L_{5}^{r}-4 L_{4}^{r}\right) \chi_{0}^{2}+\left(4 L_{6}^{r}-2 L_{4}^{r}\right) \chi_{0} \chi_{s}\right. \\
& +\left(-2 L_{5}^{r}-4 L_{4}^{r}+4 W_{8}^{r}+8 W_{6}^{r}-2 W_{5}^{r}-4 W_{4}^{r}\right) \chi_{0} \rho_{0}+\left(2 W_{6}^{r}-2 W_{4}^{r}\right) \chi_{0}|\rho| \\
& +\left(-2 L_{4}^{r}+2 W_{6}^{r}\right) \chi_{s} \rho_{0} \\
& +\left(-2 W_{5}^{r}-4 W_{4}^{r}+4 W_{8}^{\prime r}+8 W_{6}^{\prime r}\right) \rho_{0}^{2}+\left(-2 W_{4}^{r}+4 W_{6}^{\prime r}\right) \rho_{0}|\rho| \\
& \left.+\left(-4 L_{8}^{r}-8 L_{6}^{r}+4 W_{8}^{r}+8 W_{6}^{r}-2 W_{5}^{r}-4 W_{4}^{r}\right) \rho_{3}^{2}+\left(-2 L_{6}^{r}-L_{4}^{r}+2 W_{6}^{r}\right) \rho_{3}^{2} \frac{\chi_{s}}{\chi_{0}}\right] .
\end{aligned}
$$

This expression shows that the presence of the strange quark, whose mass term is not subject to any chiral twist here, foils the $\mathcal{O}(a)$-improvement at maximal twist, as expected.

The mass splitting, given by

$$
M_{\pi_{1,2}}^{2}-M_{\pi_{3}}^{2}=\frac{16}{F_{0}^{2}}\left(L_{86}^{r}-W_{86}^{r}+W_{86}^{\prime r}\right) \rho_{3}^{2},
$$

is formally equivalent to that obtained for $N_{f}=2$, taking the different Gasser-Leutwyler coefficients into account.

For the kaons we get

$$
\begin{aligned}
M_{K}^{2}= & m_{K}^{2}+\frac{m_{K}^{2}}{48 \pi^{2} F_{0}^{2}} m_{\eta}^{2} \ln \left(\frac{m_{\eta}^{2}}{\Lambda^{2}}\right) \\
& +\frac{2}{F_{0}^{2}}\left[\left(2 L_{8}^{r}+8 L_{6}^{r}-L_{5}^{r}-4 L_{4}^{r}\right) \chi_{0}^{2}+\left(4 L_{8}^{r}+16 L_{6}^{r}-2 L_{5}^{r}-6 L_{4}^{r}\right) \chi_{0} \chi_{s}\right. \\
& +\left(2 L_{8}^{r}+4 L_{6}^{r}-L_{5}^{r}-2 L_{4}^{r}\right) \chi_{s}^{2} \\
& +\left(-L_{5}^{r}-4 L_{4}^{r}+2 W_{8}^{r}+8 W_{6}^{r}-W_{5}^{r}-4 W_{4}^{r}\right) \chi_{0} \rho_{0} \\
& +\left(-L_{5}^{r}-4 L_{4}^{r}+2 W_{8}^{r}+6 W_{6}^{r}-W_{5}^{r}-2 W_{4}^{r}\right) \chi_{0}|\rho| \\
& +\left(-L_{5}^{r}-2 L_{4}^{r}+2 W_{8}^{r}+6 W_{6}^{r}-W_{5}^{r}-4 W_{4}^{r}\right) \chi_{s} \rho_{0} \\
& +\left(-L_{5}^{r}-2 L_{4}^{r}+2 W_{8}^{r}+4 W_{6}^{r}-W_{5}^{r}-2 W_{4}^{r}\right) \chi_{s}|\rho| \\
& +\left(-2 W_{5}^{r}-6 W_{4}^{r}+4 W_{8}^{\prime r}+12 W_{6}^{\prime r}\right) \rho_{0}\left(\rho_{0}+|\rho|\right) \\
& +\left(-4 L_{8}^{r}-8 L_{6}^{r}+4 W_{8}^{r}+8 W_{6}^{r}-2 W_{5}^{r}-6 W_{4}^{r}+4 W_{6}^{\prime r}\right) \rho_{3}^{2} \\
& \left.+\left(-2 L_{8}^{r}-6 L_{6}^{r}+L_{4}^{r}+2 W_{8}^{r}+6 W_{6}^{r}-W_{5}^{r}-4 W_{4}^{r}\right) \rho_{3}^{2} \frac{\chi_{s}}{\chi_{0}}\right]
\end{aligned}
$$


and for the $\eta$-mass

$$
\begin{aligned}
M_{\eta}^{2}= & m_{\eta}^{2}+\frac{m_{\eta}^{2}}{48 \pi^{2} F_{0}^{2}}\left[3 m_{K}^{2} \ln \left(\frac{m_{K}^{2}}{\Lambda^{2}}\right)-2 m_{\eta}^{2} \ln \left(\frac{m_{\eta}^{2}}{\Lambda^{2}}\right)\right] \\
& +\frac{m_{\pi}^{2}}{96 \pi^{2} F_{0}^{2}}\left[m_{\eta}^{2} \ln \left(\frac{m_{\eta}^{2}}{\Lambda^{2}}\right)-3 m_{\pi}^{2} \ln \left(\frac{m_{\pi}^{2}}{\Lambda^{2}}\right)+2 m_{K}^{2} \ln \left(\frac{m_{K}^{2}}{\Lambda^{2}}\right)\right] \\
& +\frac{4}{9 F_{0}^{2}}\left[\left(12 L_{8}^{r}+24 L_{7}^{r}+24 L_{6}^{r}-2 L_{5}^{r}-12 L_{4}^{r}\right) \chi_{0}^{2}\right. \\
& +\left(-48 L_{7}^{r}+60 L_{6}^{r}-8 L_{5}^{r}-30 L_{4}^{r}\right) \chi_{0} \chi_{s}+\left(24 L_{8}^{r}+24 L_{7}^{r}+24 L_{6}^{r}-8 L_{5}^{r}-12 L_{4}^{r}\right) \chi_{s}^{2} \\
& +\left(-2 L_{5}^{r}-12 L_{4}^{r}+12 W_{8}^{r}+24 W_{7}^{r}+24 W_{6}^{r}-2 W_{5}^{r}-12 W_{4}^{r}\right) \chi_{0} \rho_{0} \\
& +\left(-4 L_{5}^{r}-24 L_{4}^{r}-24 W_{7}^{r}+30 W_{6}^{r}-4 W_{5}^{r}-6 W_{4}^{r}\right) \chi_{0}|\rho| \\
& +\left(-4 L_{5}^{r}-6 L_{4}^{r}-24 W_{7}^{r}+30 W_{6}^{r}-4 W_{5}^{r}-24 W_{4}^{r}\right) \chi_{s} \rho_{0} \\
& +\left(-8 L_{5}^{r}-12 L_{4}^{r}+24 W_{8}^{r}+24 W_{7}^{r}+24 W_{6}^{r}-8 W_{5}^{r}-12 W_{4}^{r}\right) \chi_{s}|\rho| \\
& +\left(-10 W_{5}^{r}-24 W_{4}^{r}+36 W_{8}^{\prime r}+48 W_{7}^{\prime r}+48 W_{6}^{\prime r}\right) \rho_{0}^{2} \\
& +\left(-8 W_{5}^{r}-30 W_{4}^{r}-48 W_{7}^{\prime r}+60 W_{6}^{\prime r}\right) \rho_{0}|\rho|+\left(-24 L_{8}^{r}-24 L_{7}^{r}-24 L_{6}^{r}\right. \\
& \left.+24 W_{8}^{r}+24 W_{7}^{r}+24 W_{6}^{r}-10 W_{5}^{r}-24 W_{4}^{r}+12 W_{8}^{\prime r}+24 W_{7}^{\prime r}+24 W_{6}^{\prime r}\right) \rho_{3}^{2} \\
& \left.+\left(24 L_{7}^{r}-30 L_{6}^{r}+9 L_{4}^{r}-24 W_{7}^{r}+30 W_{6}^{r}-4 W_{5}^{r}-24 W_{4}^{r}\right) \rho_{3}^{2} \frac{\chi_{s}}{\chi_{0}}\right] .
\end{aligned}
$$

In addition to the masses we determine the decay constants for the pseudoscalar meson octet. The results are for the pions

$$
\begin{aligned}
\frac{F_{\pi}}{F_{0}}=1 & -\frac{1}{32 \pi^{2} F_{0}^{2}}\left[2 m_{\pi}^{2} \ln \left(\frac{m_{\pi}^{2}}{\Lambda^{2}}\right)+m_{K}^{2} \ln \left(\frac{m_{K}^{2}}{\Lambda^{2}}\right)\right] \\
& +\frac{4}{F_{0}^{2}}\left[L_{5}^{r} \chi_{0}+L_{4}^{r}\left(\chi_{s}+2 \chi_{0}\right)+W_{5}^{r} \rho_{0}+W_{4}^{r}\left(|\rho|+2 \rho_{0}\right)\right]
\end{aligned}
$$

for the kaons

$$
\begin{aligned}
\frac{F_{K}}{F_{0}}=1 & -\frac{3}{128 \pi^{2} F_{0}^{2}}\left[m_{\pi}^{2} \ln \left(\frac{m_{\pi}^{2}}{\Lambda^{2}}\right)+2 m_{K}^{2} \ln \left(\frac{m_{K}^{2}}{\Lambda^{2}}\right)+m_{\eta}^{2} \ln \left(\frac{m_{\eta}^{2}}{\Lambda^{2}}\right)\right] \\
& +\frac{2}{F_{0}^{2}}\left[L_{5}^{r}\left(\chi_{0}+\chi_{s}\right)+2 L_{4}^{r}\left(\chi_{s}+2 \chi_{0}\right)+W_{5}^{r}\left(|\rho|+\rho_{0}\right)+2 W_{4}^{r}\left(|\rho|+2 \rho_{0}\right)\right],
\end{aligned}
$$

and for the $\eta$ meson

$$
\begin{aligned}
\frac{F_{\eta}}{F_{0}}=1 & -\frac{3}{32 \pi^{2} F_{0}^{2}} m_{K}^{2} \ln \left(\frac{m_{K}^{2}}{\Lambda^{2}}\right) \\
& +\frac{4}{3 F_{0}^{2}}\left[L_{5}^{r}\left(2 \chi_{s}+\chi_{0}\right)+3 L_{4}^{r}\left(\chi_{s}+2 \chi_{0}\right)+W_{5}^{r}\left(2|\rho|+\rho_{0}\right)+3 W_{4}^{r}\left(|\rho|+2 \rho_{0}\right)\right] .
\end{aligned}
$$

In the limit of equal quark masses for all flavours, $\chi_{s}=\chi_{0}$, and vanishing twist these expressions coincide to

$$
\frac{F_{\pi}}{F_{0}}=1-\frac{N_{f}}{32 \pi^{2} F_{0}^{2}}\left[m_{\pi}^{2} \ln \left(\frac{m_{\pi}^{2}}{\Lambda^{2}}\right)\right]+\frac{4}{F_{0}^{2}}\left[L_{54}^{r} \chi_{0}+W_{54}^{r} \rho_{0}\right],
$$


recovering the result of [14].

As a check, in the continuum limit, $\rho=0$, our formulae for $N_{f}=3$ go over into those of [10.

The expressions given here can be used in the analysis of numerical results from unquenched simulations of twisted mass lattice QCD with two or three flavours. Fitting the data for masses and decay constants with the chiral perturbation formulae will allow to extract numerical values for the low energy coefficients [3, 25].

We thank I. Montvay, F. Farchioni and P. Hofmann for discussions.

\section{References}

[1] F. Farchioni, R. Frezzotti, K. Jansen, I. Montvay, G.C. Rossi, E.E. Scholz, A. Shindler, N. Ukita, C. Urbach and I. Wetzorke, Eur. Phys. J. C 39 (2005) 421.

[2] F. Farchioni, K. Jansen, I. Montvay, E.E. Scholz, L. Scorzato, A. Shindler, N. Ukita, C. Urbach and I. Wetzorke, Eur. Phys. J. C 42 (2005) 73.

[3] F. Farchioni et al., hep-lat/0512017.

[4] R. Frezzotti, P.A. Grassi, S. Sint and P. Weisz, Nucl. Phys. B (Proc. Suppl.) 83 (2000) 941; JHEP 0108 (2001) 058.

[5] R. Frezzotti, Nucl. Phys. B (Proc. Suppl.) 119 (2003) 140.

[6] R. Frezzotti and G.C. Rossi, JHEP 0408 (2004) 007.

[7] A. Shindler, PoS (LAT2005) 014, hep-lat/0511002.

[8] S. Weinberg, Physica A 96 (1979) 327.

[9] J. Gasser and H. Leutwyler, Ann. Phys. 158 (1984) 142.

[10] J. Gasser and H. Leutwyler, Nucl. Phys. B 250 (1985) 465.

[11] H. Wittig, Nucl. Phys. B (Proc. Suppl.) 119 (2003) 59.

[12] S. Sharpe and R. Singleton, Phys. Rev. D 58 (1998) 074501.

[13] W. Lee and S. Sharpe, Nucl. Phys. B (Proc. Suppl.) 73 (1999) 240.

[14] G. Rupak and N. Shoresh, Phys. Rev. D 66 (2002) 054503.

[15] S. Aoki, Phys. Rev. D 68 (2003) 054508.

[16] O. Bär, G. Rupak and N. Shoresh, Phys. Rev. D 70 (2004) 034508. 
[17] G. Münster and C. Schmidt, Europhys. Lett. 66 (2004) 652.

[18] G. Münster, C. Schmidt and E.E. Scholz, Europhys. Lett. 68 (2004) 639.

[19] G. Münster, JHEP 09 (2004) 035.

[20] L. Scorzato, Eur. Phys. J. C 37 (2004) 445.

[21] S. Sharpe and J.M.S. Wu, Phys. Rev. D 70 (2004) 094029.

[22] S. Aoki and O. Bär, Phys. Rev. D 70 (2004) 116011.

[23] S. Sharpe and J.M.S. Wu, Phys. Rev. D 71 (2005) 074501.

[24] S. Sharpe, Phys. Rev. D 72 (2005) 074510.

[25] F. Farchioni et al., PoS (LAT2005) 072, hep-lat/0509131, and work in progress.

[26] S. Aoki, O. Bär, S. Takeda and T. Ishikawa, Phys. Rev. D 73 (2006) 014511.

[27] A.M. Abdel-Rehim, R. Lewis, R.M. Woloshyn and J.M.S. Wu, hep-lat/0601036.

[28] A. Walker-Loud and J.M.S. Wu, Phys. Rev. D 72 (2005) 014506.

[29] R. Frezzotti and G.C. Rossi, Nucl. Phys. B (Proc. Suppl.) 128 (2004) 193.

[30] R. Frezzotti, Nucl. Phys. B (Proc. Suppl.) 140 (2005) 134. 